\title{
Pour une anthropologie de la ville et dans la ville : questions de méthodes
}

\section{Alain Hayot}

\section{Q OpenEdition}

1 Journals

\section{Édition électronique}

URL : https://journals.openedition.org/remi/2646

DOI : $10.4000 /$ remi.2646

ISSN : $1777-5418$

Éditeur

Université de Poitiers

\section{Édition imprimée}

Date de publication : 12 décembre 2002

Pagination : 93-105

ISBN : 2-911627-32-6

ISSN : 0765-0752

\section{Référence électronique}

Alain Hayot, "Pour une anthropologie de la ville et dans la ville : questions de méthodes », Revue européenne des migrations internationales [En ligne], vol. 18 - $n^{\circ} 3$ | 2002, mis en ligne le 09 juin 2006, consulté le 14 avril 2022. URL : http://journals.openedition.org/remi/2646 ; DOI : https://doi.org/ 10.4000/remi.2646

Ce document a été généré automatiquement le 14 avril 2022.

(c) Université de Poitiers 


\title{
Pour une anthropologie de la ville et dans la ville : questions de méthodes
}

\author{
Alain Hayot
}

1 Cet article se veut un exposé problématique de la capacité de l'ethnologie à comprendre l'espace urbain contemporain, en particulier les variations culturelles et les frontières symboliques qui lui donnent du sens. On notera que l'anthropologie urbaine est en plein essor et pour un chercheur qui a débuté dans cette discipline (Carreno, Hayot et Lesme, 1974), à l'époque où triomphait la macrosociologie urbaine, il y a là quelques motifs de satisfaction. Encore est-il nécessaire de bien s'entendre sur la portée et le sens d'une telle démarche. On a en effet trop souvent l'habitude de présenter l'intérêt de l'ethnologue pour la ville au pire comme une intrusion dans un monde de la modernité et de l'altérité, réservée au sociologue, au mieux comme un complément à la sociologie, l'ethnologie se chargeant d'étudier les phénomènes culturels de résistance au changement. Or si l'intervention ethnologique dans le domaine urbain a un intérêt, c'est celui d'avoir contribué à élargir la question de savoir qui produit la ville en en posant une autre : comment est habitée la ville.

2 Donner à lire la ville et les formes dont elle se dote; mettre en lumière les modes d'habiter, d'occupation et d'appropriation de l'espace urbain; étudier les formes de la sociabilité, de la cohabitation aux différents types de centralité ; analyser les modes de transition de l'espace privé à l'espace public, de l'espace résidentiel à celui du travail ; repérer les formes de perdurance et de recomposition des configurations spatiales et des identités locales (cités, quartiers, communes, mégapoles...) ; définir enfin ce qui mérite d'être élevé au rang de rituels contemporains et appelle à une recherche sur les raisons de l'émergence du phénomène et ses modes de fonctionnement (les supporters et les compétitions sportives, les fans de rock et les grands concerts, les grands rassemblements populaires et festifs notamment d'inspiration militante...).

3 Cette énumération qui ne prétend aucunement à l'exhaustivité ouvre un champ de recherche encore trop peu exploré. Il s'agit de connaître les formes urbaines contemporaines de la territorialisation des pratiques et des rapports sociaux en sachant qu'ils supposent une territorialisation faite de frontière sociale, fonctionnelle 
et symbolique, de ségrégation et d'homogénéisation, de désorganisation et de recomposition sociale, d'aliénation et d'invention culturelle, d'anomie et de recomposition identitaire. Il s'agit, enfin, de le faire impérativement dans une perspective cumulative qu'appelle de ses vœux Marcel Roncayolo (1990).

4 C'est à ce prix que l'ethnologie urbaine peut acquérir quelque intérêt: en se plaçant délibérément dans une perspective fondamentale, historique et anthropologique, dégageant les grandes tendances actuelles de l'urbanité, mode dominant pour une très longue période semble-t-il, de la territorialisation des sociétés et des établissements humains.

$5 \quad$ Fonder une telle démarche exige de se situer par rapport aux trois références majeures de la recherche urbaine en sciences sociales aujourd'hui : la sociologie urbaine des années 1970 dont nous essaierons de montrer qu'elle ne mérite pas plus l'opprobre radicale et le rejet définitif dont elle est l'objet actuellement, que l'excès d'honneur qu'elle connût à l'époque; l'École de Chicago des années vingt, dominante dans le paysage de l'anthropologie urbaine hexagonale et dont la (re)découverte depuis une dizaine d'années (Joseph et Grafmeyer, 1990) a eu le mérite de relancer la réflexion urbaine, mais qui exige une appropriation plus critique que celle qui prévaut actuellement.

6 Par ailleurs, il existe toujours depuis plusieurs années maintenant en France une ethnologie dans la ville qui privilégie l'étude des identités territoriales et / ou ethniques, des formes de la cohabitation à l'échelle d'unités résidentielles restreintes mais qui a encore des difficultés à intégrer la dimension urbaine des pratiques sociales et des représentations symboliques saisies à l'échelle de la ville dans toute son amplitude.

La sociologie urbaine des années soixante-dix

7 Prenant avec juste raison le contre-pied de l'urbanisme opérationnel coupable d'avoir défiguré la ville, cette sociologie urbaine dont la préoccupation essentielle était de mettre l'urbain en question (Castells, 1970) va le définir comme un simple lieu d'effectuation des politiques étatiques, un support passif de la reproduction du capital, de son pouvoir politique et de la force de travail.

8 Fonder l'émergence d'une ethnologie urbaine française entre autres, sur la critique de cette sociologie ne doit cependant pas nous empêcher de lui reconnaître des mérites que l'on a tort de sous-estimer aujourd'hui : M. Castells, J. Lojkine, C. Topalov et bien d'autres, ont contribué d'une manière décisive à sortir la recherche urbaine du technocratisme au sein duquel une "science » de l'aménagement l'avait enfermée, en réduisant les problèmes posés par les villes à des questions purement techniques et fonctionnelles. Là où l'aménagement ne voyait que distorsion ou désordre à réguler, la sociologie urbaine des années soixante-dix y verra un ordre social et un ordonnateur, l'État. Là où les aménageurs ne verront que la conjonction anarchique de forces aveugles à canaliser, cette sociologie va s'attacher à démonter le jeu complexe des déterminants économiques et politiques à l'œuvre dans les transformations qui s'opèrent.

9 En ces temps d'urbanisation généralisée où l'espace est un enjeu majeur de société, l'urbanisme peut en effet difficilement se définir comme ayant une simple fonction régulatrice: développant les travaux de $\mathrm{H}$. Lefebvre, cette sociologie a montré concrètement à quel point l'urbanisme est un acte politique, opérant des choix, 
reproduisant les divisions sociales ; à quel point il peut être aussi un lieu de compromis entre forces sociales, voire de résistance à l'ordre social dominant.

Cette sociologie a présenté un avantage considérable : elle a contribué à éclairer la recherche en sciences sociales sur les enjeux réels de la question urbaine. Mais elle est tombée dans un certain nombre d'écueils que l'on peut résumer de la manière suivante : à vouloir à toutes forces définir un ordre unique et généralisable, elle a développé une approche frontale et globale de l'État, comme s'il s'agissait d'une structure homogène, dépourvue de contradictions internes, d'un bloc central dépourvu de toute démultiplication; à définir l'urbain comme la reproduction localisée de processus politico-économiques globaux, elle a nié toute réalité à la ville dans ses dimensions concrètes : l'urbain c'est aussi des habitants qui instaurent entre eux des relations et des rapports sociaux s'inscrivant dans des pratiques, dans un territoire, dans une histoire, dans des logiques morphologiques, architecturales et sociales.

11 La ville est produite quotidiennement par ceux qui la vivent dans une dialectique complexe, souvent contradictoire avec une autre forme de production issue des déterminations de caractère économique, étatique qui, décentralisation aidant, mettent en mouvement des structures de plus en plus localisées et proches, théoriquement, des citadins. Et c'est cette dialectique qui peut le mieux définir un concept, pourtant largement utilisé dans les années soixante-dix, mais d'un point de vue réducteur, celui de politique urbaine.

12 Cependant, à aucun moment, l'intérêt ne se porte sur les habitants, sur leur mode d'occupation et d'appropriation de l'espace public et privé de leur ville, sur les pratiques résidentielles, les formes de consommation, de sociabilité, de territorialisation.

13 Affirmer cela, est-ce pour autant sombrer dans l'écologisme et le culturalisme, que dénonçait dans La question urbaine Manuel Castells, confondant dans la même critique Henri Lefebvre et l'École de Chicago (1970) ? Bien évidemment non. La suite a montré qu'il y avait derrière cela un débat sur l'espace et le statut du territoire dans la définition même des rapports sociaux.

14 C'est l'objet de notre troisième remarque : la critique de Manuel Castells en effet nous enferme dans une fausse alternative qui fonctionne tel un jeu de miroirs, renvoyant la même image mais inversée : ou l'espace urbain comme simple support d'effectuation des rapports sociaux ou l'espace comme producteur de pratiques et de représentations. Mais le débat sur l'espace n'en n'est plus là et cette opposition entre ce que JeanCharles Depaule appelait « les théories de la cristallisation et de la condensation », est largement dépassé (1980). «Il est toujours dangereux », écrit M. Roncayolo se référant à $\mathrm{H}$. Lefebvre, « de considérer le territoire comme simple support alors que le définir en termes de construction territoriale rappelle une dimension fondamentale des sciences sociales (1990). Et des architectes aussi ont pris conscience de la complexité et de la dialecticité des rapports espace et société : c'est Christian Devillers qui le résume le mieux: "Il est d'usage de considérer les phénomènes spatiaux comme de simples conséquences du développement social et économique... Or si l'espace est jusqu'à un certain point le produit des rapports sociaux, il a par rapport à eux une existence spécifique car il est en même temps le lieu préexistant dans lequel ils s'effectuent et l'un de leurs enjeux les plus importants. Il faut dès lors (...) chercher dans l'histoire des rapports sociaux les informations nécessaires à la compréhension des espaces et celle 
qui informe la connaissance (...) des rapports sociaux par l'analyse des configurations spatiales » (1981).

L'ethnologie, depuis son origine, met l'accent sur cette démarche. Dès Marcel Mauss et L'essai sur les variations saisonnières des Eskimos (1969) ou Les classifications primitives (1966), on sait que tout établissement humain est à la fois organisation sociale et unité territoriale. Avec Claude Lévi-Strauss, l'espace, l'organisation territoriale du village tribal, apparaît comme une référence essentielle dans les processus de reproduction sociale (1958). André Leroi-Gourhan nous montre à quel point la pratique et l'adhésion à un territoire dépendent à la fois de la connaissance que le groupe en a, et du temps qu'il passe à l'acquérir et à construire un rapport techno-économique et symbolique avec lui (1964).

Contrairement à la sociologie qui a souvent traité des relations ou des rapports sociaux comme s'ils se déroulaient sur un coussin d'air, ou bien considérait qu'un espace n'était qualifié que par ce que les hommes y effectuaient ${ }^{1}$, la tradition ethnologique ne sépare jamais l'étude des rapports entre les hommes de celle des rapports des hommes à leur milieu.

La ville moderne échapperait-elle à une telle démarche? Sa dimension, la désorganisation sociale qu'elle connaît, l'individualisation des comportements que chacun croit déceler, provoqueraient-ils une crise irréversible de la territorialité ? l'École de Chicago, paradoxalement, propose à cette question des réponses non univoques.

L'école de Chicago

18 Il convient de constater que, malgré l'effort effectué par Halbwachs dans les années trente, et quelques références éparses (par exemple C. Lévi-Strauss dans Anthropologie structurale), L'École de Chicago est globalement restée méconnue en France, y compris au sens strict du terme, puisque ses principaux travaux vont attendre longtemps avant d'être traduits. Si René Duchac (1969) lui consacre une thèse à la fin des années soixante, il faut attendre la fin de la décennie suivante pour qu'un courant se crée en France en faveur de cette école, que les textes essentiels soient publiés, que des enquêtes soient menées sur la base des problématiques initiées par ses principaux théoriciens. Entre temps, il y avait eu la critique de M. Castells (1970). Mais tout semble se dérouler aujourd'hui comme si nous étions passés d'une critique réductrice des problématiques de Chicago à une adoption enthousiaste et sans débat critique réel de ses résultats, et peut-être surtout de ses présupposés théoriques et méthodologiques.

Critique réductrice : cette école a été taxée d'écologisme (survalorisation du rôle du cadre écologique dans la structuration des comportements sociaux) et de culturalisme (définition d'une culture urbaine issue de ce cadre, possédant sa propre logique, une logique quelque peu ahistorique et autonome au sein de la formation sociale). Ces deux critiques occultent néanmoins l'immense matériau empirique et méthodologique recueilli sur la ville et la société américaines de l'entre-deux-guerres. Elle nie l'existence d'une démarche, à l'opposé certes d'une analyse de la production globale de la ville, mais qui introduit une ethnologie des citadins riche en observations et qui rend compte de l'épaisseur des rapports sociaux. Elle passe à côté d'un certain nombre d'apports à la connaissance de la réalité urbaine contemporaine que l'on peut difficilement ne pas reconnaître aux théoriciens américains de l'entre-deux-guerres : 
20 - celui d'avoir mis l'accent sur la territorialisation des processus sociaux, territorialisation que la recherche urbaine a longtemps occultée, sauf dans ses dimensions de sociologie politique ;

21 - l'accent mis également sur le fait ethnique dans l'analyse des relations sociales, en particulier à travers la question du migrant, aspect également occulté par la sociologie urbaine, parce que posé comme alternatif à une analyse en termes de rapports sociaux de classe ;

22 - l'accent mis enfin sur une méthodologie de type qualitatif aujourd'hui largement utilisée pour analyser les processus sociaux, les pratiques et représentations des individus: biographies, récits de vie, monographies de quartiers, d'institutions, de groupes sociaux et/ou ethniques, observation d'espaces urbains supports intenses de pratiques de sociabilité, analyse de réseaux, formes contemporaines d'urbanité.

23 On ne peut cependant réduire la (re)découverte de l'École de Chicago à une faiblesse théorique et méthodologique de la recherche française dans ces domaines. L'appel à cette école ne remplit pas seulement un vide, il participe certes de manière contradictoire, à une évolution qui travaille la société française et les sciences sociales elles-mêmes. Il faut en effet remarquer que cette (re)découverte est corrélative de l'émergence de deux faits : à l'échelle de la société française, la reterritorialisation des politiques sociales et institutionnelles et l'apparition du local comme enjeu fondamental de régulation et de contrôle social; sur le plan des sciences sociales, l'épanouissement d'une sociologie interactionniste où les rapports sociaux cèdent la place aux relations interindividuelles saisies dans une quotidienneté immédiate, possédant sa propre logique.

24 Le retour de l'acteur, le local comme enjeu décisif, voilà un cadre idéal pour que Park, Wirth et Burgess fassent école. Ils ont en effet réussi ce paradoxe étonnant de concilier d'une part, une conception biologique du territoire, conçu comme un agrégat de communautés aux strictes règles internes se disputant leur «aire naturelle » d'implantation et, d'autre part une référence majeure à une théorie du sujet libre que Gérard Althabe critique de la manière suivante: "La sociabilité urbaine s'impose aux individus, mais contient en elle du fait de la pluralité des situations, la liberté de l'individu qui se met à distance et négocie ses choix. Les individus évoluent dans un cadre préétabli » (1984). La définition de ce cadre comme donnée naturelle fait l'économie de l'analyse de sa construction socio-historique.

25 Affirmer un désaccord avec les présupposés théoriques de l'École de Chicago, n'interdit pas l'intégration d'un certain nombre d'acquis méthodologiques et de résultats empiriques dans une perspective cumulative.

26 En revanche, fonder et développer une ethnologie urbaine non comme outil de régulation sociale, mais comme instrument de connaissance des formes contemporaines de la territorialisation des rapports sociaux, exige un point de vue critique à l'égard de l'École de Chicago et de ses successeurs, sur des questions aussi cardinales que sa référence constante à la biologie, son rejet de l'histoire, ses tendances à la modélisation et à l'universalisation de "situations » observées dans une société et un espace-temps précis. La seule condition exigible étant que les deux démarches, intégration et confrontation critiques ne paraissent possibles que dans le cadre de recherches concrètes, sur des terrains et des objets urbains d'aujourd'hui, autorisant la comparaison des méthodes et des résultats. Il s'agit là d'une démarche modeste, 
ponctuelle, mais somme toute plus efficace sur le long terme. C'est la tâche que doit s'assigner l'anthropologie urbaine contemporaine.

Une ethnologie dans la ville

L'ethnologie urbaine française (Gutwirth, 1987, 1988; Althabe, 1992), de par ses méthodes et ses objets de recherche, a souvent privilégié l'échelle locale et les unités territoriales restreintes. De même sa filiation avec l'ethnologie classique qui a porté sur des terrains «exotiques » et sur les sociétés traditionnelles, essentiellement en milieu rural, l'a amenée à privilégier l'étude de communautés ou de collectivités homogènes présentant des caractéristiques de spécificité, de stabilité et de relations sociales denses. La société locale est, alors, considérée comme une structure autonome, un système d'institution et d'activités qui forment un ensemble intégré. Il n'y a pas de rupture, mais communication continue, entre les relations de parenté, de voisinage et d'inter-connaissance. L'ethnologie a privilégié l'échelle locale afin de rompre avec les approches macro-sociologiques et afin de prendre en compte la diversité et la singularité du lieu évitant, ainsi, de le fondre dans une globalité homogénéisante.

Si cette approche a été plus ou moins aisée à mettre en œuvre dans les sociétés traditionnelles ou dans les communautés rurales, elle a, par contre, éprouvé quelques difficultés à s'appliquer en milieu urbain. La démarche ethnologique a été confrontée à la spécificité de la ville définie comme espace de diversité, de l'anonymat, de la fragmentation des lieux et des activités, de la rupture entre l'espace public et l'espace privé, de l'instabilité des relations, de la mobilité (résidentielle, professionnelle...), de brassages sociaux multiples, de la faiblesse de la fonction intégratrice du territoire d'habiter. Le citadin dans ces conditions se trouve pris dans un jeu complexe de rôles, de statuts et d'appartenances qui débordent le cadre étroit de son espace résidentiel ou de son quartier.

29 L'ethnologie urbaine devait aussi intégrer dans son champ d'analyse les différentes transformations socio-économiques qui ont affecté la société dans son ensemble: l'industrialisation; l'importance de la mobilité ; l'urbanisation à grande échelle des années 1960-1970 (le zoning, l'implantation des grands ensembles résidentiels telles que les H.L.M. sans articulation aux quartiers ou aux noyaux villageois existants); la tendance au repli domestique ; le déclin des quartiers ouvriers et populaires ainsi que des formes de sociabilité et des relations sociales qui y prévalaient et qui s'ancraient dans certains lieux publics (cafés, cercles, chorales, diverses associations, activités militantes, fêtes patronales, bal...) ; intégration au logement de nouvelles formes de confort qui ont réduit ou ont fait disparaître certains lieux publics de rencontre et d'échanges tels que lavoirs, bains-douches, fontaines...

On peut dire que l'ethnologie urbaine a opté plus pour une ethnologie dans la ville que pour une ethnologie de la ville. En privilégiant cette approche elle a porté son attention sur « l'identitaire ", les univers à forte sociabilité, et sur les relations sociales fortement territorialisée dans l'espace public. C'est notamment le cas des minorités ethniques et plus largement de ce qu'on désigne du terme de "village urbain ». La métaphore villageoise vise à qualifier une manière d'être en ville cumulative de différents traits : homogénéité du peuplement et des modes de vie; relations sociales centrées sur le quartier; densité des interconnaissances dans un espace local restreint; importance des réseaux d'entraide et d'échange qui sont aussi des moyens d'exercice du contrôle social lié au voisinage; lien social dans le quartier renforcé par le fait concret de la 
proximité et de la familiarisation ; maîtrise de l'espace public par les habitants à travers sa fréquentation quotidienne.

Pour une anthropologie de la ville et dans la ville : échelles territoriales et frontières symboliques

31 Nous envisageons les territoires dans l'espace urbain non comme un agrégat naturel de populations aux caractéristiques communes, une manifestation d'un "vouloir vivre ensemble » mais plutôt comme le résultat d'un processus socio-historique qui peut déboucher sur une conscience identitaire collective, un sentiment d'appartenance à un groupe, à un lieu, voire à un "style" pour reprendre une notion chère à LeroiGourhan.

32 Si la ville est faite de mobilité, elle est d'abord «la somme de nombreuses parties, de quartiers, de secteurs très différents, dont les caractéristiques formelles et sociologiques sont elles-mêmes très différentes » (Rossi, 1981). L'auteur précise que ces secteurs renvoient à des moments de la ville, une temporalité qui se définit à partir de trois caractéristiques qui fournissent autant de champs d'investigation :

33 - une fonction urbaine issue d'une histoire et de rapports sociaux spécifiques, définissant une organisation institutionnelle ou non du territoire, des activités économiques propres, les catégories sociales qui y vivent ;

34 - une forme urbaine, matérialité du territoire cumulative de tous les contenus pour reprendre l'expression d'Henri Lefebvre dans la Révolution urbaine (1970); elle se construit donc en même temps que son paysage social, histoire à laquelle elle participe activement au point de peser lourdement sur les modes de reproduction sociale: culture locale, formes de l'appropriation de l'espace, mémoire collective et individuelle...;

35 - une vie collective, en termes de sociabilité et de relations de voisinage ou plus simplement de comportements communs, de références communes à un système de valeurs, à une même conception de l'appropriation de l'espace.

36 À partir de là, l'émergence d'une identité locale semble dépendre de plusieurs conditions : une inscription socio-spatiale dans la durée; une certaine similitude dans les conditions sociales d'existence, supposant l'existence ou la construction d'une certaine proximité culturelle; une relative homogénéité morphologique du territoire définissant sinon des typologies architecturales communes, du moins des limites nettes, reconnues comme telles.

37 Analyser les territoires dans la ville sur la base d'une telle problématique appelle cependant quelques précautions méthodologiques sur les notions centrales de quartier, d'échelle, de frontière et enfin d'ethnicité.

38 La notion de quartier exprime un véritable paradoxe. C'est au moment où le quartier, en tant que configuration territoriale, échelle de référence identitaire et d'appartenance sociale et résidentielle est en voie de décomposition et de recomposition que l'on éprouve le besoin de recourir à elle: soit comme cadre géographique des actions publiques territorialisées (D.S.Q; D.S.U) soit comme cadre de recherche des sciences sociales. Le quartier, doté de qualités identitaires ou communautaires supposées, serait une des réponses à certains problèmes sociaux ou à la déliquescence du lien social, d'où l'idée récente de proximité réinvestie par les politiques publiques d'emploi, d'insertion, etc. L'image du quartier est alors invoquée, souvent, pour stigmatiser le mode de vie urbain qui serait synonyme d'anonymat, d'aliénation, de vide sociale, d'anomie, de 
dissolution des solidarités et de fragmentation des liens sociaux. Dans cette perspective, plusieurs recherches (Althabe, 1984) ont fait du quartier le lieu d'une vie sociale harmonieuse marquée par l'intensité des relations sociales, par des formes de solidarité et d'entraide faites de partage de valeurs et d'une culture locale forte et homogène. Le quartier est, alors, défini, à la fois par le sens commun et le savoir scientifique, comme valeur et comme essence qui cumulent tous les traits d'un donné naturel. Il présenterait, selon cette approche, les caractéristiques d'un isolat ou d'un microcosme autonome par rapport à l'ensemble urbain où il s'insère. De ce point de vue, le quartier présente des traits du mythe.

En outre, on confond quartier et communauté. En présupposant l'autonomie du quartier, on tend à en faire le réceptacle de toutes les activités (économiques, commerciales, professionnelles, etc.) et des relations sociales des habitants. Ces derniers n'inscrivent évidemment pas toutes les manifestations et les expressions de leur existence urbaine dans le cadre étroit du quartier, mais intègrent aussi d'autres espaces de la ville : leurs réseaux de relations et d'activités articulent différentes unités et échelles territoriales de la ville.

La question qui se pose immédiatement est celle de la définition des limites du quartier. Les habitants interrogés lors de nos enquêtes à Marseille éprouvent une certaine difficulté pour fixer les limites de leur quartier. Ils en ont une vision subjective et affective qui se réfère à leur pratique quotidienne. Le quartier peut renvoyer, dans ce cas, soit à leur voisinage restreint, soit à leur itinéraire quotidien constitué d'arrêts chez le commerçant, au marché, au bistrot, etc. En croisant les différentes limites que proposent les habitants, il est difficile d'en déduire une délimitation et une définition synthétique et stable. L'image retenue est celle de limites territoriales variables et changeantes qui peuvent aller de l'unité de voisinage à une unité spatiale plus large (Arrif et Hayot, 2001). À la différence des limites administratives (l'INSEE regroupe différentes unités résidentielles : le noyau villageois, les cités H.L.M., les lotissements, etc.) les habitants proposent un quartier plus éclaté, plus fragmenté et plus réduit. Dans ce cas, le quartier peut se restreindre au noyau villageois ou bien aux cités suivant le processus d'identification par différenciation ou opposition. « Perçu comme un bloc de l'extérieur, le quartier est en fait marqué par son hétérogénéité culturelle et sociale interne " (Segalen, 1990). Il nous faut donc refuser toutes généralisations: la «banlieue », l'« espace de l'exclusion», le «Bronx» ou à l'inverse, « le village dans la ville ». Il est donc nécessaire de travailler en même temps sur ce qui unifie et sur ce qui différencie socialement, culturellement et symboliquement.

41 La notion d'échelles spatiales, appliquée à l'espace urbain, permet d'appréhender le territoire de la ville non pas comme une unité spatiale homogène mais comme un espace composé, formé de la sédimentation et de l'articulation de ses multiples sousterritoires. Cette manière d'aborder l'urbain permet d'envisager la multiplicité des rapports aux territoires qui s'établissent dans la ville sans les figer ni surdéterminer le local. La prise en compte des échelles spatiales des pratiques - sociales et territoriales - induit des déplacements de perspectives qui, à chaque fois, donnent un éclairage nouveau selon que l'on considère le groupe social au niveau de l'unité résidentielle et du quartier ou bien au niveau des autres unités territoriales proches ou lointaines ou encore au niveau de l'ensemble urbain.

Ces « glissements » et emboîtements des territoires, et le déplacement de perspectives qu'ils nécessitent, sont le lieu de tension entre différentes polarités caractéristiques de 
l'urbain: continuité / discontinuité ; localisme / mobilité ; pratiques centripètes / pratiques centrifuges ; proximité / distance.

Chaque échelle territoriale renvoie, du point de vue des représentations, à une certaine image qui peut être de l'ordre de l'expérience ou bien du stéréotype. C'est ainsi qu'on associe à l'espace du proche, le cercle des relations familiales, de voisinage ou d'interconnaissance et les sentiments de protection, de maîtrise, d'ancrage et à l'espace $\mathrm{du}$ lointain, les relations diffuses relevant de l'anonymat, de la mobilité, de l'impersonnalité, de l'étrangéité. Mais il ne faudrait pas attribuer à ces polarités des valeurs positives ou négatives absolues. C'est, au contraire, à travers le rapport singulier que nouent un individu ou un groupe avec chacune de ces échelles territoriales et à partir du sens qu'ils donnent à ces relations et aux pratiques qui les actualisent, qu'il faudrait rechercher les valeurs qui leur sont attribuées.

L'exemple de la mobilité des jeunes résidant dans les périphéries urbaines illustre bien la nécessité de relativiser les valeurs associées à l'espace proche ou lointain. Car tout en s'identifiant à leur lieu de résidence, les jeunes - notamment issus de l'immigration inscrivent leurs pratiques de mobilité dans des réseaux qui «transversalisent » les appartenances territoriales.

La notion d'échelle a été inscrite, dès le début de nos recherches et de façon explicite, dans notre approche de la question des frontières dans l'espace urbain marseillais. Nous avons refusé de porter notre regard uniquement sur la macro-frontière entre les Quartiers-Nord et les Quartiers-Sud de Marseille, et nous avons affiné ce découpage afin d'intégrer, dans une perspective comparative, les micro-frontières à l'échelle de chaque quartier, faisant partie de nos terrains d'enquête, pour ensuite les articuler aux autres paliers - inter-quartiers et ville dans son ensemble. Cette variation et ce croisement d'échelles nous a permis de répondre à notre question initiale : comment est habitée la ville? de quelle manière les différenciations culturelles, les formes de sociabilité, les appartenances, les pratiques se territorialisent de façon singulière dans un espace urbain souvent décrit comme le lieu de l'indifférenciation, de l'homogénéisation et de la perte des identités (Arrif et Hayot, 2001).

46 La notion de frontière appliquée au territoire urbain, telle que nous la concevons, ne s'apparente pas à une frontière géographique ou à une barrière infranchissable délimitant des territoires autonomes, totalement discontinus sans aucune communication (sociale, urbaine, équipement, voirie, etc.). De même, la frontière n'a pas un contenu ni une forme figés telles que les limites administratives ou les limites d'un État-Nation qui requièrent une réglementation, une régulation et un contrôle institutionnel des passages d'un territoire à un autre.

47 La frontière ne correspond pas à une rupture radicale de communication et de relations, mais témoigne de la tension et du conflit qui les animent. Elle est, fondamentalement, une construction passée et à l'œuvre, sociale et symbolique qui peut, par ailleurs, s'appuyer sur des éléments territoriaux, architecturaux et topographiques; donc sur une matérialité qui donne une certaine consistance à son existence et l'inscrit dans l'ordre de l'évidence. Comme une seconde nature qui provoque une sorte d'amnésie des conditions mêmes de sa genèse et des logiques de son instrumentation dans le champ des relations sociales.

48 Insister sur la dimension " constructive » de la notion de frontière, c'est lui donner une acception problématique, ouverte; c'est, aussi, l'inscrire au centre des luttes et des conflits de catégorisation, de désignation et de qualification des groupes résidentiels 
concernés et de leur territoire d'habiter. Elle relève de plusieurs ordres servant de référents au discours dont elle est l'objet. Ces ordres peuvent être constitués de différents éléments hétérogènes, auxquels le discours sur les frontières donnent une certaine cohérence pratique, relevant soit de l'identité, soit du partage des traits communs liés à l'histoire, à l'origine sociale, ethnique, culturelle, soit à une relative homogénéité morphologique du territoire définissant sinon des typologies architecturales (cabanons, village-rue, pavillons, cités H.L.M. - barre, tours -, etc.) communes, du moins des limites nettes reconnues telles (exemple des limites paroissiales); à partir desquels un ordre de proximité et de distance, de ressemblance et de différence sera établi. Le territoire urbain, en question, ne correspond pas seulement à sa matérialité spatiale, mais renvoie à des formes de territorialités dans la mesure où il est approprié, qualifié, nommé et marqué par une présence qui se veut singulière et spécifique.

C'est cette volonté de singulariser le territoire et de lui donner une valeur quasi emblématique qui pose la question de l'identité et des appartenances territoriales. Ces identités territoriales, dont les registres et les sources de légitimation sont multiples (mémoire, ancienneté, appartenance à un groupe dominant, autochtonie, identité nationale, valeurs), cristallisent la tension entre différentes polarités: l'allogène/ l'exogène; le légitime/l'illégitime du lieu; «Nous»/»Eux»; proximité spatiale/ distance sociale ; proximité sociale/distance morale, etc.

Sur nos terrains marseillais, nous avons pris la mesure de l'importance du terme d'ethnicisation, pris dans une acception dynamique, dans la construction symbolique des frontières spatiales et territoriales. Et l'on soulignera que l'usage de cette notion n'a de validité que dans la mesure où elle s'articule à une parole habitante, à un imaginaire et à des représentations dont la préoccupation centrale est de produire des barrières, de signifier des distances sociales, culturelles et territoriales à travers le langage propre à la stigmatisation, au stéréotype, au racisme.

51 Les liens sociaux au sein de nos terrains marseillais sont marqués par la tension, l'affrontement et, également, par des pratiques d'évitement et de stigmatisation d'une partie des habitants. Les populations les plus visées et vis-à-vis desquelles on veut se différencier - et dont la présence est considérée comme illégitime et perturbatrice de l'ordre normatif du quartier - sont les populations immigrées ou celles qui sont considérées comme étrangères à son histoire. Ainsi, la nationalité n'est souvent pas le référent majeur puisque sont concernés, également, des Français d'origine étrangère récente ou de confessions différentes, des ressortissants des DOM-TOM, des PiedsNoirs. Le consensus observé autour de ces populations, traités comme ensemble homogène et spécifique, est un des vecteurs majeurs de production identitaire des groupes dits de « souche».

La stigmatisation et la construction de groupes allogènes comme pôle négatif de référence s'inscrit dans un processus complexe de construction sociale de la figure de "l'étranger». Cette construction sociale de l'étranger, proche spatialement mais maintenu dans une distance sociale et symbolique, est véhiculée à travers différentes formes et expressions dont celle de l'ethnicisation des relations sociales. On tend ainsi à enfermer un groupe, celui des immigrés, dans un registre identitaire et culturel unique et homogène référant au partage, supposé, par tous ses membres d'une même origine et d'une même appartenance ethnique. Un des effets d'une telle représentation est la 
radicalisation de l'altérité de ce groupe et de son extériorité au quartier. Même si sa présence est ancienne et même s'il partage certains référents culturels.

Conclusion

53 Le foisonnement actuel de travaux ayant l'espace urbain, la ville comme terrain d'enquête, le croisement sur un même objet de problématiques, de concepts, de méthodes, issues de l'ethnologie classique, de l'anthropologie urbaine anglo-saxonne ou de disciplines voisines comme la sociologie, la géographie, l'histoire et l'architecture méritait que l'on pose des questions épistémologiques quant à la pertinence et la légitimité du bricolage théorique auquel nous sommes nombreux à procéder. La ville et ses territoires donnent à voir des frontières socio-symboliques sédimentées à travers le temps ou plus récentes. Ainsi l'espace social urbain se fragmente en quartiers, cités, banlieues, espaces publics divers, en aires ségrégatives contemporaines qui forment autant d'espaces de représentations, se fractionnant et s'emboîtant selon les circonstances et les situations en plus ou moins grandes unités d'appartenances et de pratiques.

Les politiques publiques dans leur recherche d'un mode de régulation sociale alternatif aux formes historiques d'intégration des quartiers populaires ont tendance à favoriser les regroupements communautaires, les associations à base ethnique, l'objectif avoué étant de s'appuyer sur des médiateurs sociaux et culturels. Mais paradoxalement cela participe de la désignation ethnique d'une population «à problèmes " et de la définition ethnique d'un espace tout aussi problématique. Dans cette perspective, l'ethnicité est moins de l'ordre de la culture, définie de façon essentialiste, qu'une catégorisation symbolique socialement construite. On le voit, la ville d'aujourd'hui mobilise, à biens des égards, une part essentielle des ressources sociales et symboliques des sociétés contemporaines.

\section{BIBLIOGRAPHIE}

ALTHABE Gérard, FABRE Daniel et LENCLUD Gérard Éds., (1992) Vers une ethnologie du présent, coll. Ethnologie de la France, Paris, M.S.H., cahier $n^{\circ} 7$.

ALTHABE Gérard (1984), L'ethnologie urbaine : ses tendances actuelles, Terrain, $n^{\circ} 3$ « Ethnologie urbaine ", Paris.

ARRIF Abdelmajid et HAYOT Alain (2001) Les territoires dans la ville. Frontières sociales et symboliques à Marseille, in Christian Bromberger et A. Morel Éds., Limites floues et frontières vives, Éd. de la MSH, Paris.

BLEITRACH Danielle, LOJKINE Jean, OARY E., DELACROIX R. et MAHIEU Christian. (1981) Classe ouvrière et social-démocratie : Lille et Marseille, Paris, Éditions sociales.

BURGESS Ernest and BOGUE Donald (1964) Research in Urban Society : a long view, In Contributions to Urban Sociology, University of Chicago Press. 
CARRENO J.A., HAYOT Alain et LESME Francis, (1974) Ethnologie d'un centre urbain : le quartier de la Porte d'Aix à Marseille, Paris, Musée de l'Homme.

CASTELLS Manuel (1970) La question urbaine, Paris, Maspero.

CASTELLS Manuel (1972) Luttes urbaines, Paris, Maspero.

DEPAULE Jean-Charles, PANERAI Philippe, et al. (1980) Éléments d'analyse urbaine, Bruxelles, A.A.M. DEVILLERS Christian et HUET Bernard (1981) Le Creusot, Naissance et développement d'une ville industrielle, 1782-1914, Seyssel, Champ Vallon.

DUCHAC René (1969) La sociologie des migrations aux U.S.A., Paris, La Haye, Mouton.

GUTWIRTH Georges et PETONNET Colette $(1987,1988)$ Chemins de la ville et Ethnologues dans la ville, éd. du C.T.H.S.

HALBWACHS Maurice (1972) Classes sociales et morphologie, Paris, Minuit.

JOSEPH Isaac et GRAFFMEYER Yves (1990) L'École de Chicago, Naissance de l'écologie urbaine, Paris, Aubier.

LEFEBVRE Henri (1970) La révolution urbaine, Paris, Gallimard.

LEROI-GOURHAN andré (1964) Le Geste et la parole : Techniques et langage, Paris, Albin Michel.

LEVI-STRAUSS Claude (1958) Anthropologie structurale, Paris, Plon.

MAUSS Marcel et DURKHEIM Emile (1966) De quelques formes primitives de classifications. Contribution à l'étude des représentations collectives, In Euvres II, Mouton.

MAUSS Marcel (1969) L'essai sur les variations saisonnières des Eskimos, In Euvres, Tomes I, II, III, Éds Victor Karady, Paris, Minuit, 2160 p.

PARK Robert E. (1952) Human Communities. The City and Human Ecology, Free Press, New York.

POUTIGNAT Philippe et STREIFF-FENART Jocelyne (1995) Théories de l'ethnicité, Paris, P.U.F., coll. "Le Sociologue ».

RONCAYOLO Marcel (1990) La ville et ses territoires, Paris, Gallimard, coll. Essais.

ROSSI Aldo (1981) L'architecture de la ville, Paris, L'Équerre.

SEGALEN Martine (1990) Nanterriens, des familles dans la ville : une ethnologie de l'identité, Toulouse, Presses Universitaires du Mirail.

TOPALOV Christian (1974) Les promoteurs immobiliers, Paris, Mouton.

WIEVIORKA Michel (1993) La démocratie à l'épreuve : Nationalisme, populisme, ethnicité, Paris, La Découverte, coll. Essais.

WIRTH Louis (1980) Le Ghetto, Grenoble, PUG

\section{NOTES}

1. -À quelques remarquables exceptions près dont les plus notables sont $\mathrm{M}$. Halbwachs (1972) et l'œuvre de H. Lefebvre qui considère qu'étudier les rapports sociaux en dehors de leur espace est " pur idéalisme ». 
INDEX

Mots-clés : anthropologie, concept, épistémologie, frontière, méthodologie, sociologie urbaine, territoire, ville

\section{AUTEUR}

\section{ALAIN HAYOT}

Professeur d'ethnologie, École d'Architecture de Marseille, 184 Avenue de Luminy, 13009 Marseille. 